\title{
ANALYTICAL SOLUTION FOR TRANSIENT ONE- DIMENSIONAL COUETTE FLOW CONSIDERING CONSTANT AND TIME-DEPENDENT PRESSURE GRADIENTS
}

\author{
A. A.Mendiburu ${ }^{a}$, \\ L. R. Carroccib ${ }^{\mathrm{b}}$, \\ andJ. A. Carvalho ${ }^{c}$ \\ ${ }^{\mathrm{a}}$ Universidade Estadual Paulista \\ "Júlio Mesquita Filho" \\ Faculdade de Engenharia de Guaratinguetá \\ Departamento de Energia \\ pos11026@ feg.unesp.br \\ ${ }^{\mathrm{b}, \mathrm{c}}$ UniversidadeEstadual Paulista \\ "Júlio Mesquita Filho" \\ Faculdade de Engenharia de Guaratinguetá \\ Departamento de Energia \\ Av. Ariberto Pereira Cunha, 333 \\ CEP.12516-410 \\ Guaratinguetá, SP, Brazil \\ Carrocci@feg.unesp.br \\ joao@feg.unep.br

\section{ABSTRACT} \\ This paperaims to determine the velocity profile, in transient state, for a \\ parallel incompressible flow known as Couette flow. The Navier-Stokes \\ equations were applied upon this flow. Analytical solutions, based in Fourier \\ series and integral transforms, were obtained for the one-dimensional \\ transient Couette flow, taking into account constant and time-dependent \\ pressure gradients acting on the fluid since the same instant when the plate \\ starts it's movement. Taking advantage of the orthogonality and \\ superposition properties solutions were foundfor both considered cases \\ Considering a time-dependent pressure gradient, it was found a general \\ solution for the Couette flow for a particular time function. It was found that \\ the solution for a time-dependent pressure gradient includes the solutions for \\ a zero pressure gradient and for a constant pressure gradient. \\ Keywords: Couette flow, Transient, Parallel plates, Incompressible fluid.

\section{NOMENCLATURE} \\ b constant pressure gradient, $\mathrm{Pa} / \mathrm{m}$ \\ $\mathrm{D}_{\mathrm{n}} \quad$ Fourier series constant, $\mathrm{m} / \mathrm{s}$ \\ $\mathrm{L}$ parallel plates distance, $\mathrm{m}$ \\ $\mathrm{N}_{\mathrm{n}} \quad$ Fourier series normalization integral, $\mathrm{m}$ \\ $\mathrm{P}$ pressure, $\mathrm{Pa}$ \\ fluid velocity in $\mathrm{x}$ direction, $\mathrm{m} / \mathrm{s}$ \\ $\mathrm{U}$ upper plate velocity, $\mathrm{m} / \mathrm{s}$ \\ $\mathrm{x}, \mathrm{y}, \mathrm{z}$ orthogonal components \\ $\rho \quad$ density, $\mathrm{kg} / \mathrm{m}^{3}$ \\ $v \quad$ kinematic viscosity, $\mathrm{m}^{2} / \mathrm{s}$ \\ $\emptyset$ homogeneous partial differential equation \\ characteristic function \\ $\lambda \quad$ constant for separation of variables \\ Subscripts \\ n Fourier series term number \\ p steady state velocity profile component \\ $\mathrm{t}$ transient state velocity profile component

\section{INTRODUCTION}

\section{Couette flow between parallel plates} \\ Consider a Newtonian fluid with density $\rho$ and \\ kinematic viscosity $v$ bounded by two infinite \\ parallel plates separated by a distance $\mathrm{L}$, as shown in \\ figure 1 . The liquid and the plates are initially at rest. \\ At time $t=0^{+}$the upper plate starts moving with \\ steady velocity $U$ while the lower plate is \\ heldstationary. At this same instant a pressure \\ gradient, acting in the direction of flow, appears.
}

$\mathrm{t}$ time, $\mathrm{s}$

Greek symbols 


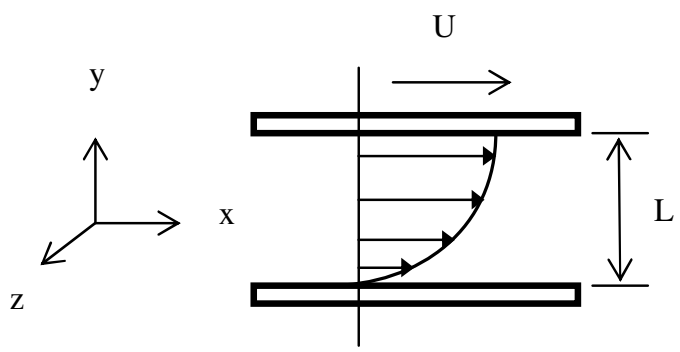

Figure 1. Couette flow between parallel infinite plates.

The problem is to determine the velocity profile in transient state for this flow. Shames (1992) provides a solution for the steady state velocity profile and pressure gradient he reached the solution by using separation of variables which is applicable in the steady state case. A more complex solution involving the transient state Couette flow is given by Papanastasiou et al. (2000), this solution is achieved by considering zero pressure gradient, in this case separation of variables and Fourier series were used, the same transient state Couette flow was solved by Graebel(2007) by writing the velocity in dimensionless form as a function of density, time, viscosity, space coordinate and plate velocity, and in doing so transforming the partial differential equation into an ordinary differential equation, again in this solution the pressure gradient was taken to be zero. This work aims to find velocity profiles for the cases of constant pressure gradient and time-dependent pressure gradient, the solutions for the two considered cases can be found by applying Fourier series and Fourier integral transforms both techniques have been successfully applied to solve transient heat conduction problems by Kakaç and Yener (1985).

The idea of a time-dependent pressure gradient was proposed by Riley and Drazin (2006) as a mean to find general solutions for one-dimensional flows.

\section{Differential equation, initial and boundary conditions}

To model this problem we use the NavierStokes equations. Assuming an incompressible Newtonian one-dimensional flow in the $\mathrm{x}$ direction we obtain the following differential equation.

$$
v \frac{\partial^{2} u}{\partial y^{2}}-\frac{\partial u}{\partial t}=\frac{1}{\rho} \frac{\partial P}{\partial x}
$$

For the given problem, the partial derivative of the pressure in the $y$ direction is zero; therefor the most general pressure function must have the following form.

$$
P=-\rho[f(t)+x g(t)]
$$

Tanking the pressure partial derivative in the $\mathrm{x}$ direction Eq. (1) becomes.

$$
v \frac{\partial^{2} u}{\partial y^{2}}-\frac{\partial u}{\partial t}=-g(t)
$$

For the initial condition we assume that there is no flow before $\mathrm{t}=\mathrm{0}^{+}$and for the boundary conditions we assume the non-slip condition which implies that the fluid in contact with the plates has the same velocity as them.

$$
\begin{aligned}
& u(0, t)=0 \\
& u(L, t)=U \\
& u(y, 0)=0
\end{aligned}
$$

As can be seen the second condition, Eq. (5), is a non-homogeneous boundary condition. We will deal with this non-homogeneity using the superposition property as follows.

$$
u(y, t)=u_{p}(y)-u_{t}(y, t)
$$

Here the first term of the left-hand side holds for the steady state component of the velocity profile and the second term for the transient state component.

\section{Pressure Gradient}

We will find the velocity profiles for a constant and a time-dependent pressure gradient represented by Eq. (8) and Eq. (9) respectively.

$$
\begin{gathered}
\frac{\partial P}{\partial x}=b \\
\frac{\partial P}{\partial x}=J b+(1-J) b e^{-(n \pi)^{2} \frac{v t}{L^{2}}} \quad \text { for } \quad 0 \leq J \leq 1
\end{gathered}
$$

The $\mathrm{b}$ and $\mathrm{J}$ factors are both constants. The main idea for representing the pressure gradient as it was represented in Eq. (9) is because we expect that the time-dependent component of the pressure gradient will eventually vanish and only the constant component will remain. We will further discuss this function's factors once we have solved the partial differential equation Eq. (1).

\section{Steadystate component for constant pressure gradient.}

For the steady state component we have the following differential equation. 


$$
\frac{\partial^{2} u}{\partial y^{2}}=\frac{1}{v \rho} b
$$

Applying a constant pressure gradient and using Eq. (4) and Eq. (5) as the boundary conditions this differential equation yields the steady state component of the velocity profile.

$$
u_{p}(y)=\frac{b}{2 v \rho}\left(y^{2}-L y\right)+\frac{U}{L} y
$$

\section{Transient state component for constant pressure} gradient.

Replacing Eq. (7) and (11) into Eq. (1), Eq. (4), Eq. (5) and Eq. (6) we obtain the differential equation, initial and boundary conditions for the transient state component of the velocity profile.

$$
\begin{aligned}
& \frac{\partial^{2} u_{t}}{\partial y^{2}}-\frac{1}{v} \frac{\partial u_{t}}{\partial t}=0 \\
& u(0, t)=0 \\
& u(L, t)=0 \\
& u(y, 0)=\frac{b}{2 v \rho}\left(y^{2}-L y\right)+\frac{U}{L} y
\end{aligned}
$$

We have now a homogeneous differential equation with homogeneous initial and boundary conditions which can be solved using Fourier series. Eq. (12) can be solved applying separation of variables, where we havechosen an adequate constant to equal the two resulting differential equations.

$$
\frac{d T}{d t} \frac{1}{v T}=\frac{d^{2} Y}{d y^{2}} \frac{1}{Y}=-\frac{\lambda^{2}}{L^{2}}
$$

Eq. (16) has the following general solution.

$$
u_{t}(y, t)=C e^{-\frac{\lambda^{2}}{L^{2}} v t}\left(A \sin \frac{\lambda}{L} y+B \cos \frac{\lambda}{L} y\right)
$$

The constant $\mathrm{B}$ is found to be zero while it is impossible to express constants $\mathrm{A}$ and $\mathrm{C}$ using simple functions; however they can be represented using Fourier series. The characteristic function for this system is.

$$
\phi_{n}(y)=\sin \frac{n \pi}{L}=\sin \frac{\lambda}{L}
$$

The normalization integral and the constants for the Fourier series solution are determined by the following equations.

$$
\begin{gathered}
N_{n}=\int_{0}^{L} \sin ^{2} \frac{n \pi}{L} y d y=\frac{L}{2} \\
D_{n}=\frac{1}{N_{n}} \int_{0}^{L}\left[\frac{b}{2 v \rho}\left(y^{2}-L y+\frac{U}{L} y\right] \sin \frac{n \pi}{L} y d y\right. \\
=\frac{b L^{2}}{2 v \rho}\left[\frac{2}{(n \pi)^{3}}\left[(-1)^{n}-1\right]\right]+\frac{2 U}{n \pi}(-1)^{n+1}
\end{gathered}
$$

The transient state component is then given by

$$
\begin{aligned}
& u_{t}(y, t)=\sum_{n=1}^{\infty}\left(\left\{\frac{b L^{2}}{2 v \rho}\left[\frac{2}{(n \pi)^{3}}\left[(-1)^{n}-1\right]\right]\right.\right. \\
& \left.+\frac{2 U}{n \pi}(-1)^{n+1}\right\} e^{-(n \pi)^{2} \frac{v t}{L^{2}}} \sin n \pi \frac{y}{L}
\end{aligned}
$$

\section{Velocity profile for Constant Pressure Gradient.}

Replacing Eq. (11) and Eq. (21) into Eq. (7) we obtain the velocity profile for the Couette flow in transient state and with a constant pressure gradient applied. Afterwards we have made the necessary arrangements to obtain this profile as a function of dimensionless factors. The function obtained is shown in Eq. (22).

$$
\begin{aligned}
& \frac{u(y, t)}{U}=\frac{b L^{2}}{2 U v \rho}\left[\left(\frac{y}{L}\right)^{2}-\frac{y}{L}\right]+\frac{y}{L} \\
& -\sum_{n=1}^{\infty}\left(\left\{\frac{b L^{2}}{U v \rho}\left[\frac{2}{(n \pi)^{3}}\left[(-1)^{n}-1\right]\right]\right.\right. \\
& \left.+\frac{2}{n \pi}(-1)^{n+1}\right\} e^{-(n \pi)^{2} \frac{v t}{L^{2}}} \sin n \pi \frac{y}{L}
\end{aligned}
$$

Steady state component for time-dependent pressure gradient.

In this case we need to solve the following differential equation.

$$
\frac{\partial^{2} u_{p}}{\partial y^{2}}=\frac{1}{v \rho}(\mathrm{Jb})
$$


This is practically the same as Eq. (10), but here the constant pressure gradient is being multiplied by $\mathrm{J}$. The boundary conditions are the same we used to solve Eq. (10) the resulting steady state component is.

$$
u_{p}(y)=J \frac{b}{2 v \rho}\left(y^{2}-L y\right)+\frac{U}{L} y
$$

\section{Transient state component for time-dependent pressure gradient.}

Replacing Eq. (9) and Eq. (24) into Eq. (1) Eq. (4), Eq. (5) and Eq. (6) we obtain the differential equation, initial and boundary conditions for the transient state component of the velocity profile.

$$
\begin{aligned}
\frac{\partial^{2} u_{t}}{\partial y^{2}}-\frac{1}{v} \frac{\partial u_{t}}{\partial t} & =\frac{\partial^{2} u_{p}}{\partial y^{2}}-\frac{1}{v \rho} g(t) \\
u_{t}(0, t) & =0 \\
u_{t}(L, t) & =0 \\
u_{t}(y, t) & =u_{p}(y)
\end{aligned}
$$

This system can be solved applying integral transforms; this procedure aim to vanish the derivatives in the y direction from Eq. (25). We apply the following transform.

$$
\bar{u}_{t}\left(\lambda_{n}, t\right)=\int_{0}^{L} k\left(\lambda_{n}, y\right) u_{t}(y, t) d y
$$

This transform's inversion formula is.

$$
u_{t}(y, t)=\sum_{n=1}^{\infty} k\left(\lambda_{n}, y\right) \bar{u}_{t}\left(\lambda_{n}, t\right)
$$

The factor $k\left(\lambda_{n}, y\right)$ is known as the kernel of the transform and it is determined by solving the system formed by Eq. (25), Eq. (26), Eq. (27) and Eq. (28) without the non-homogeneous terms, this procedure will yield the same characteristic function and normalization integral as the ones considered in Eq. (18) and (19) respectively. For the kernel we have

$$
k\left(\lambda_{n}, y\right)=\frac{\phi_{n}(y)}{\sqrt{N_{n}}}=\sqrt{\frac{2}{L}} \sin \frac{n \pi}{L} y
$$

The next step is to multiply Eq. (1) by $k\left(\lambda_{n}, y\right)$ and then integrate from zero to $\mathrm{L}$.

$$
\begin{aligned}
& \int_{0}^{L} k\left(\lambda_{n}, y\right) \frac{\partial^{2} u_{t}}{\partial y^{2}} d y-\frac{1}{v} \int_{0}^{L} k\left(\lambda_{n}, y\right) \frac{\partial u_{t}}{\partial t} d y \\
& =\int_{0}^{L} k\left(\lambda_{n}, y\right) h(y, t) d y
\end{aligned}
$$

where

$$
\begin{gathered}
h\left(\lambda_{n}, t\right)=\frac{\partial^{2} u_{p}}{\partial y^{2}}-\frac{1}{v \rho} f(t) \\
=-(1-J) \frac{b}{v \rho} e^{-(n \pi)^{2} \frac{v t}{L^{2}}}
\end{gathered}
$$

For the secondterm on left-hand side of Eq. (32) the following relation can be proved

$$
\int_{0}^{L} k\left(\lambda_{n}, y\right) \frac{\partial u_{t}}{\partial t} d y=\frac{d \bar{u}_{t}}{d t}
$$

Integrating by parts the first term on the lefthand side of Eq. (32) and applying the boundary conditions we get

$$
\int_{0}^{L} k\left(\lambda_{n}, y\right) \frac{\partial^{2} u_{t}}{\partial y^{2}} d y=-\int_{0}^{L} \frac{d k}{d y} \frac{\partial u_{t}}{\partial y} d y
$$

Integrating by parts Eq. (35) we obtain

$$
\int_{0}^{L} k\left(\lambda_{n}, y\right) \frac{\partial^{2} u_{t}}{\partial y^{2}} d y=\int_{0}^{L} \frac{d^{2} k}{d y^{2}} u_{t} d y
$$

Where

$$
\frac{d^{2} k}{d y^{2}}=-\sqrt{\frac{2}{L}} \frac{(n \pi)^{2}}{L^{2}} \sin \frac{n \pi}{L} y=-\frac{(n \pi)^{2}}{L^{2}} k\left(\lambda_{n}, y\right)
$$

The right-hand side term of Eq. (32) can be expressedas

$$
\int_{0}^{L} k\left(\lambda_{n}, y\right) h(y, t) d y=\bar{h}\left(\lambda_{n}, t\right)
$$

This transform yields

$$
\begin{gathered}
\overline{\mathrm{h}}\left(\lambda_{\mathrm{n}}, \mathrm{t}\right)=(1-\mathrm{J})\left[(-1)^{\mathrm{n}}-1\right] \times \\
\frac{\mathrm{L}}{\mathrm{n} \pi} \sqrt{\frac{2}{\mathrm{~L}}} \frac{\mathrm{b}}{\mathrm{v} \rho} \mathrm{e}^{-(\mathrm{n} \pi)^{2} \frac{\mathrm{vt}}{\mathrm{L}^{2}}}
\end{gathered}
$$


The two terms of the right-hand side in Eq. (39) are multiplying each other. For the following step we need to consider the next exponential factor

$$
e^{-(n \pi)^{2} \frac{v t}{L^{2}}}
$$

Replacing Eq. (34), Eq. (36), Eq. (37) and Eq. (39) in Eq. (32), then multiplying by Eq. (40) and finally integrating from zero to $t$ we get

$$
\begin{aligned}
& \int_{0}^{t} e^{-(n \pi)^{2} \frac{v t}{L^{2}}}\left[-\frac{(n \pi)^{2}}{L^{2}} \bar{u}_{t}\left(\lambda_{n}, t\right)-\frac{1}{v} \frac{d \bar{u}_{t}}{d t}\right] d t \\
& \int_{0}^{t} e^{-(n \pi)^{2} \frac{v t}{L^{2}}}\left\{(1-J)\left[(-1)^{n}-1\right] \times\right. \\
& \left.\frac{L}{n \pi} \sqrt{\frac{2}{L}} \frac{b}{v \rho} e^{-(n \pi)^{2} \frac{v t}{L^{2}}}\right\} d t
\end{aligned}
$$

As can be seen the exponential terms in the right-hand side of Eq. (41) will cancel each other, it must also be noted that

$$
\begin{aligned}
& -\frac{1}{v} \frac{d}{d t}\left[e^{(n \pi)^{2} \frac{v t}{L^{2}}} \bar{u}_{t}\left(\lambda_{n}, t\right)\right] \\
= & e^{(n \pi)^{2} \frac{v t}{L^{2}}}\left[-\frac{(n \pi)^{2}}{L^{2}} \bar{u}_{t}\left(\lambda_{n}, t\right)-\frac{1}{v} \frac{d \bar{u}_{t}}{d t}\right]
\end{aligned}
$$

Using Eq. (42) to solve the left-hand side integral in Eq. (41) and then solving for $\bar{u}_{t}$ we get

$$
\begin{array}{r}
\bar{u}_{t}\left(\lambda_{n}, t\right)=v t e^{-(n \pi)^{2} \frac{v t}{L^{2}}}\left\{\bar{u}_{t}\left(\lambda_{n}, 0\right)\right. \\
\left.-\left[(1-J)\left[(-1)^{n}-1\right] \frac{L}{n \pi} \sqrt{\frac{2}{L}} \frac{b}{v \rho}\right]\right\}
\end{array}
$$

Here $\bar{u}_{t}\left(\lambda_{n}, 0\right)$ represents the integral transform of the initial condition, so we have

$$
\begin{gathered}
\bar{u}_{t}\left(\lambda_{n}, 0\right)=\int_{0}^{L} k\left(\lambda_{n}, y\right) u_{p}(y) d y \\
=\sqrt{\frac{2}{L}\left[\frac{2 L^{3}}{(n \pi)^{3}} \frac{J b}{2 v \rho}\left[(-1)^{n}-1\right]+\frac{U L}{n \pi}(-1)^{n+1}\right]}
\end{gathered}
$$

Replacing Eq. (44) into Eq. (43) we obtain the following function for the integral transform of thetransient state component of the velocity profile

$$
\begin{aligned}
& \bar{u}_{t}\left(\lambda_{n}, t\right)=e^{-(n \pi)^{2} \frac{v t}{L^{2}}}\left\{\sqrt{\frac{2}{L}} \frac{2 L^{3}}{(n \pi)^{3}} \frac{J b}{2 v \rho}\left[(-1)^{n}-1\right]\right. \\
& +\sqrt{\frac{2}{L} \frac{U L}{n \pi}(-1)^{n+1}} \\
& \left.-\left((1-J)\left[(-1)^{n}-1\right] \frac{L}{n \pi} \sqrt{\frac{2}{L}} \frac{b}{v \rho}\right) v t\right\}
\end{aligned}
$$

Now using the inversion formula we obtain the transient velocity profile

$$
\begin{aligned}
& \frac{\mathrm{u}_{\mathrm{t}}(\mathrm{y}, \mathrm{t})}{\mathrm{U}}=\sum_{\mathrm{n}=1}^{\infty}\left\{\sin \frac{\mathrm{n} \pi}{\mathrm{L}} \mathrm{ye} \mathrm{e}^{-(\mathrm{n} \pi)^{2} \frac{v \mathrm{t}}{\mathrm{L}^{2}}} \times\right. \\
& {\left[\mathrm{J} \frac{\mathrm{bL}}{\mathrm{Uv \rho}} \frac{2}{(\mathrm{n} \pi)^{3}}\left[(-1)^{\mathrm{n}}-1\right]+\frac{2}{\mathrm{n} \pi}(-1)^{\mathrm{n}+1}\right.} \\
& \left.\left.-\left((1-\mathrm{J}) \frac{\mathrm{bL}}{\mathrm{Uv \rho}} \frac{2}{\mathrm{n} \pi}\left[(-1)^{\mathrm{n}}-1\right]\right) \frac{v \mathrm{t}}{\mathrm{L}^{2}}\right]\right\}
\end{aligned}
$$

Here the sin and exponential terms are multiplying each other and then multiply the resulting term of the operations inside the claps.

Finally the total velocity profile can be expressed by replacing Eq. (24) and Eq. (46) into Eq. (7)

$$
\begin{aligned}
& \frac{\mathrm{u}(\mathrm{y}, \mathrm{t})}{\mathrm{U}}=\frac{\mathrm{J}}{2} \frac{\mathrm{bL}}{\mathrm{Uv} \rho}\left(\left(\frac{\mathrm{y}}{\mathrm{L}}\right)^{2}-\frac{\mathrm{y}}{\mathrm{L}}\right)+\frac{\mathrm{y}}{\mathrm{L}} \\
& -\sum_{\mathrm{n}=1}^{\infty}\left\{\sin \frac{\mathrm{n} \pi}{\mathrm{L}} \mathrm{ye} \mathrm{e}^{-(\mathrm{n} \pi)^{2} \frac{v \mathrm{t}}{\mathrm{L}^{2}}} \times\right. \\
& {\left[\mathrm{J} \frac{\mathrm{bL}}{\mathrm{Uv \rho}} \frac{2}{(\mathrm{n} \pi)^{3}}\left[(-1)^{\mathrm{n}}-1\right]+\frac{2}{\mathrm{n} \pi}(-1)^{\mathrm{n}+1}\right.} \\
& \left.\left.-\left((1-\mathrm{J}) \frac{\mathrm{bL}}{\mathrm{Uv \rho}} \frac{2}{\mathrm{n} \pi}\left[(-1)^{\mathrm{n}}-1\right]\right) \frac{v \mathrm{t}}{\mathrm{L}^{2}}\right]\right\}
\end{aligned}
$$

\section{Dimensionless parameters}

Inspecting the velocity profiles, Eq. (22) and Eq. (47), we find four dimensionless parameters, among these the most important are the following.

$$
\begin{aligned}
& \frac{b L^{2}}{U v \rho} \\
& \frac{v t}{L^{2}}
\end{aligned}
$$


The first one represents the relation between the pressure gradient and plate distance with the kinematic viscosity, density and plate velocity. The second dimensionless parameter represents the influence of the kinematic viscosity and plate distance with the time needed for the fluid to achieve steady state flow.

\section{RESULTS AND DISCUSIONS}

In this section we present some plots for the velocity profile in function of the two dimensionless parameters.

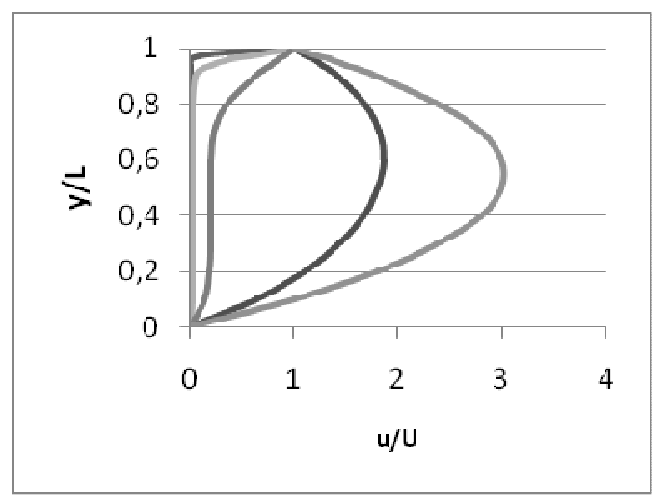

Figure 2.Velocity profile for constant pressuregradient.

$$
\frac{b L^{2}}{U v \rho}=-20 \text { and } \frac{v t}{L^{2}} \text { from } 0.0001 \text { to } 1
$$

Figures 2 and 3 were both plotted using Eq. (22). Figure 2 represents a decreasing pressure on the direction of flow while figure 3 represents an adverse pressure gradient or, in other words, an increasing pressure gradient in the direction of flow. On both cases we can observe that as the time passes the profile tends to become quasi parabolic. Both figures show a unitary horizontal displacement respect to the origin, this is due to the non-slip condition statement.

At greater values of Eq. (48) the influence of plate velocity upon the total velocity profile will be negligible. For small values of Eq. (49) the Fourier series require at least 50 terms to converge, as Eq. (49) value approaches to 1 less than 10 terms will be required.

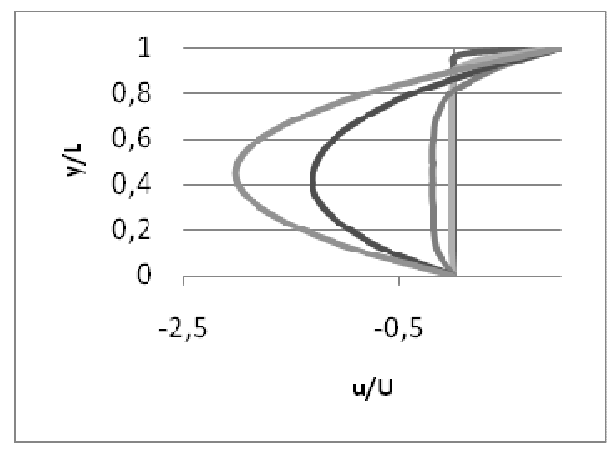

Figure 3.Velocity profile for constant pressuregradient.

$$
\frac{b L^{2}}{U v \rho}=20 \text { and } \frac{v t}{L^{2}} \text { from } 0.0001 \text { to } 1 .
$$

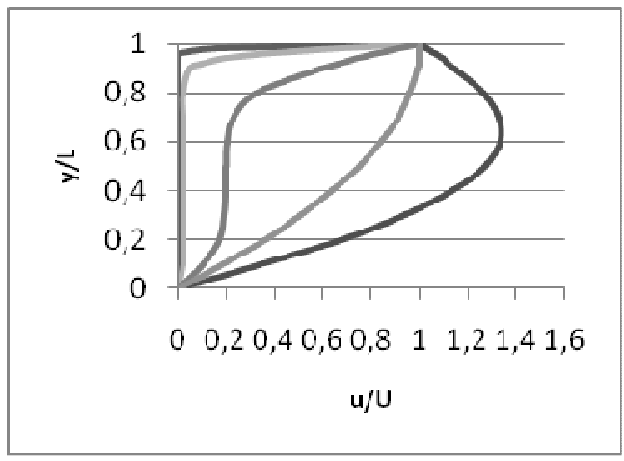

Figure 4.Velocity profile for constant pressure gradient.

$$
\frac{b L^{2}}{U v \rho}=-20, J=0.1 \text { and } \frac{v t}{L^{2}} \text { from } 0.0001 \text { to } 1 .
$$

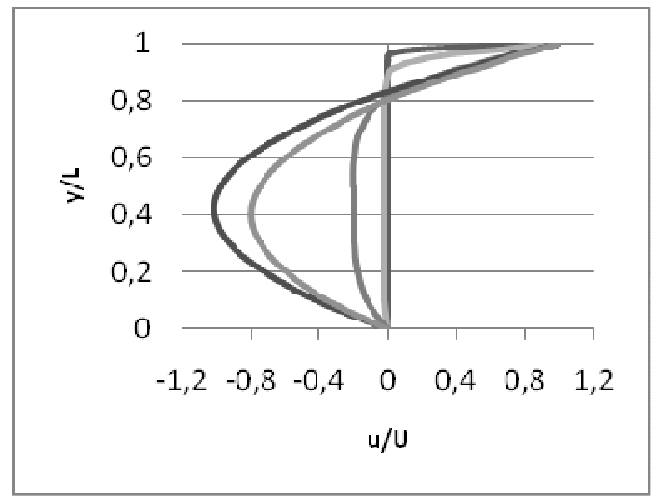

Figure 5.Velocity profile for constant pressure gradient.

$$
\frac{b L^{2}}{U v \rho}=20, J=0.1 \text { and } \frac{v t}{L^{2}} \text { from } 0.0001 \text { to } 1 \text {. }
$$

Figure 4 and figure 5 show a decreasing pressure on the flow direction and an adverse pressure gradient, bothwere plotted using Eq. (47). 
Inspecting the time-dependent function Eq. (9) it can be seen that the time-dependent component of the pressure gradient will decrease and eventually vanish as the time passes, it can also be noted that for $\mathrm{J}=1$ Eq. (47) is equal to Eq. (22). It is now obvious that the time-dependent function for the pressure gradient was chosen so it would tend to constant pressure gradient as time passes.

When the pressure gradient is small we expect the velocity profile, determined by Eq. (47) to approximate the velocity profile for zero pressure gradient, this behavior is shown in figure 6 and figure 7. Figure 8 was plotted using Eq. (47) and it shows the velocity profile when the pressure gradient is zero.

Comparing figure 2 with figure 4 and figure 3 with figure 5 , we note that the main differences between these figures are the maximumvelocity value and, when the velocity profilebecomes quasiparabolic, the perpendicular distance from the symmetry axis to any point on the parabola.

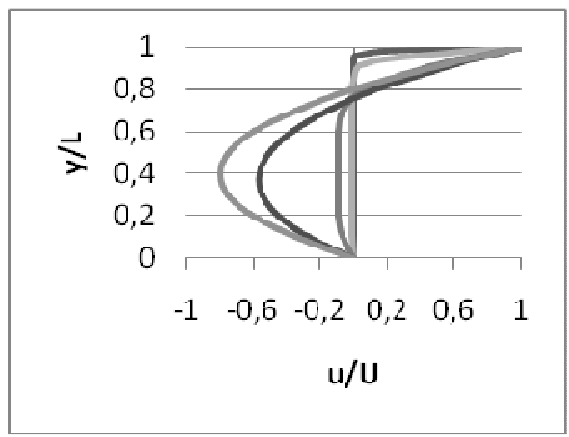

Figure 6.Velocity profile for constant pressure gradient.

$$
\frac{b L^{2}}{U v \rho}=10, J=1 \text { and } \frac{v t}{L^{2}} \text { from } 0.0001 \text { to } 1 \text {. }
$$

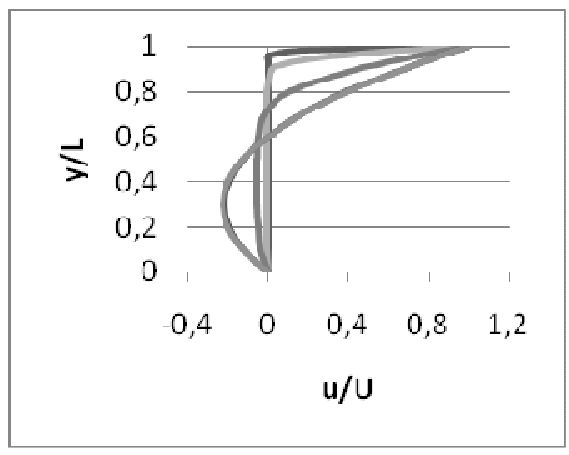

Figure 7.Velocity profile for constant pressure gradient.

$$
\frac{b L^{2}}{U v \rho}=5, J=1 \text { and } \frac{v t}{L^{2}} \text { from } 0.0001 \text { to } 1 .
$$

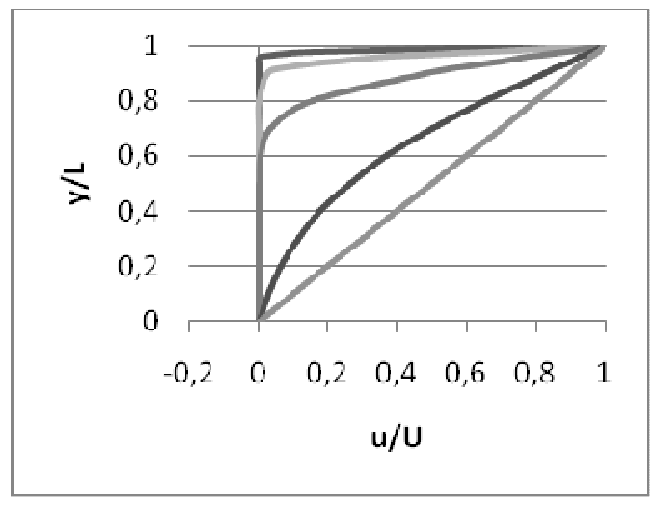

Figure 8. Velocity profilefor Zero pressure gradient.

$$
\frac{b L^{2}}{U v \rho}=0, J=1 \text { and } \frac{v t}{L^{2}} \text { from } 0.0001 \text { to } 1 \text {. }
$$

\section{CONCLUSIONS}

On the analysisof one dimensional transient Couette flow, consideration of a time-dependent pressure gradient makes possible to obtain generalsolutions for the velocity profile. In this work was considered a specific time-dependent function which led us to a solution that contains the solutions for zero pressure gradient and constant pressure gradient, as was expected.

Different time-dependent functions can be used and the same procedure applied in order to find different solutions, although it should be noted that only the last term inside the sum of Eq. (47) would substantially change.

Is important to point the fact that the velocity profiles obtained Eq. (22) and Eq. (47), are both dimensionless and depend on three parameters which are also dimensionless. Therefore, the velocity profiles found can be applied to any incompressible fluid confined between two plates separated by an arbitrary distance and for an arbitrary plate velocity as well.

\section{REFERENCES}

Graebel, W. P., 2007, Advanced Fluid Mechanics, Elsevier Book Company.

Kakaç, S., and Yener, Y., 1985, Heat Conduction, Springer Book Company.

Papanastasiou, T., and Georgiou, G., 2000, Viscous Fluid Flow, CRC Press Book Company.

Riley, N., and Drazin, P., 2006, The NavierStokes Equations a Classification of Flows and Exact Solutions, Cambridge University Press Book Company.

Shames, I., 1992, Mechanics of Fluids, McGraw-Hill Book Company.

\footnotetext{
Received: June 09, 2009

Revised: July 09, 2009

Accepted: August 09, 2009
} 